\title{
A survey on social jetlag in Japan: a nationwide, cross-sectional internet survey
}

\author{
Yoko Komada $^{1}$ (D) Isa Okajima ${ }^{2} \cdot$ Shingo Kitamura $^{3} \cdot$ Yuichi Inoue $^{4}$
}

Received: 22 April 2019 / Accepted: 18 June 2019 / Published online: 25 June 2019

(c) The Author(s) 2019

\begin{abstract}
Misalignment between an individual's circadian phase and his/her environment due to social imposition is called social jetlag (SJL). SJL has emerged as a public health concern, as it contributes to an unhealthy lifestyle and adverse health outcomes. This study aimed to elucidate the percentage of SJL in a large Japanese population using the Japanese version of the Munich ChronoType Questionnaire (MCTQ). A total of 10,000 participants responded to a web-based cross-sectional survey, and the final sample comprised 3708 participants (60.1\% male; average age 45.1 [SD 13.4] years). SJL was measured by subtracting each participant's midpoint of sleep on workdays from their midpoint of sleep on free days. The average [SD] absolute SJL (SJLabs) value was 0.91 [0.89] hours, and the percentage of participants with SLJabs of more than $1 \mathrm{~h}$ was $40.1 \%$. Our data also showed that SJLabs becomes progressively smaller with advancing age. A considerable number of participants of our Japanese population showed differences in sleep behavior between workdays and free days. Young people in particular commonly oversleep on free days to compensate for the sleep debt accumulated over the workweek because of their late chronotype.
\end{abstract}

Keywords Social jetlag $\cdot$ Chronotype $\cdot$ Misalignment $\cdot$ Munich ChronoType Questionnaire $\cdot$ Sleep

\section{Introduction}

The endogenous circadian timing system is responsible for the maintenance and coherence of physiological processes and their synchronization with environmental light-dark cycles $[1,2]$. Individuals show different stable phase alignments to the 24-h day, which are called chronotypes. Chronotypes appear to be largely determined by the genetic composition of an individual's circadian clock [3-5]. An individual may be able to choose to change their sleep timing based on social impositions, but they may not be able to shift their internal clocks in the same way, owing to their

Yoko Komada

komay@my-pharm.ac.jp

1 Liberal Arts, Meiji Pharmaceutical University, 2-522-1 Nosio, Kiyose-city, Tokyo 204-8588, Japan

2 Department of Psychological Counseling, Faculty of Humanities, Tokyo Kasei University, Tokyo, Japan

3 Department of Sleep-Wake Disorders, National Institute of Mental Health, National Center of Neurology and Psychiatry, Tokyo, Japan

4 Department of Somnology, Tokyo Medical University, Tokyo, Japan genetic background. Misalignment between an individual's circadian phase and their environment due to social imposition is called social jetlag (SJL).

SJL is a measure of the discrepancy in sleep timings between our workdays and free days $[6,7]$ and was so named owing to the similarity to travel-induced jetlag caused by taking a flight to the west (east) on Friday evening and a return flight on Monday morning. Travel-induced jetlag results in a misaligned circadian system causing temporary problems; in contrast, SJL has chronic consequences. SJL has been related to unhealthy behaviors such as smoking [6] or physical inactivity [8] and has been suggested as a risk factor for obesity [9], metabolic dysfunction that may lead to a predisposition to diabetes, atherosclerotic cardiovascular disease [10-12], and depression [13]. It has emerged as a public health concern. A recent study also indicated that SJL is negatively associated with serum brain-derived neurotrophic factor (BDNF) levels, which plays an important role in neuronal maintenance, plasticity, and neurogenesis [14].

A number of sociodemographic and behavioral correlates of SJL have been reported. SJL is associated with technology access in the bedroom as well as with internet use in the hour before bed [15]. Furthermore, when 
commuting times become longer, SJL increases [16]. Certain stimulants such as nicotine and caffeine have been shown to be associated with SJL $[6,16]$, although directional causality cannot be inferred.

Data collected in Europe using the Munich ChronoType Questionnaire (MCTQ), which enquires about the sleep behavior on workdays and free days separately, has revealed that nearly two-thirds of the working population show at least $1 \mathrm{~h}$ of SJL, and that one-third of the working population suffers from an SJL of $2 \mathrm{~h}$ or more [3]. In another study conducted in a nationally representative sample of adults in the UK, the average SJL duration was 36 min in participants with less than $7 \mathrm{~h}$ of sleep [17]. The study using the American Time Use Survey revealed that as much as $60 \%$ of the US population has a late chronotype, and probably suffers from at least some amount of SJL and sleep loss [18]. However, nationwide MCTQ surveys have not been conducted in Asia. In a Japanese sample, the bedtime was late and the mean sleep duration was shorter compared to other countries [19, 20], indicating that short sleep on weekdays results in sleep debt, which is relieved by longer weekend sleep. It is hypothesized that a considerable percentage of the Japanese population experience SJL to compensate for sleep debt, and SJL is affected by sex, age, and chronotype, similar to results from surveys in central Europe, the UK and the US. Therefore, this study aimed to elucidate the percentage of SJL in a large Japanese population using the Japanese version of MCTQ [21].

\section{Materials and methods}

\section{Participants and ethics}

A web-based cross-sectional survey was conducted among registered participants of a survey company in Japan. Participants who were matched for age, sex, and resident area to a representative Japanese sample were randomly invited to participate in the survey. Initially, a total of 10,000 participants responded to the questionnaire.

We excluded those who provided invalid answers, aged under 20 or over 70 , who did not have a regular work schedule, who reported sleep times on free days with the use of alarm clocks, a requirement for determining chronotype according to the MCTQ [2], and who worked as shift workers. The final sample comprised 3708 participants $(60.1 \%$ male, average age: 45.1 [SD 13.4] years old, Fig. 1).

This study was approved by the ethics committee of Tokyo Medical University (no. 3307). The study participants provided informed consent through the consent agreeing/ disagreeing web page.

\section{Measurements}

We obtained sociodemographic and lifestyle characteristics of the participants, that is, age, sex, height, weight, smoking status (yes/no), alcohol consumption (yes/no), physical activity of more than 30 min on 2 days per week continuously for more than 1 year (yes/no), disease under treatment (yes/no), duration using display devices such as a smartphone, television, or personal computer after
Fig. 1 Flowchart of sample selection and geographical locations of participants

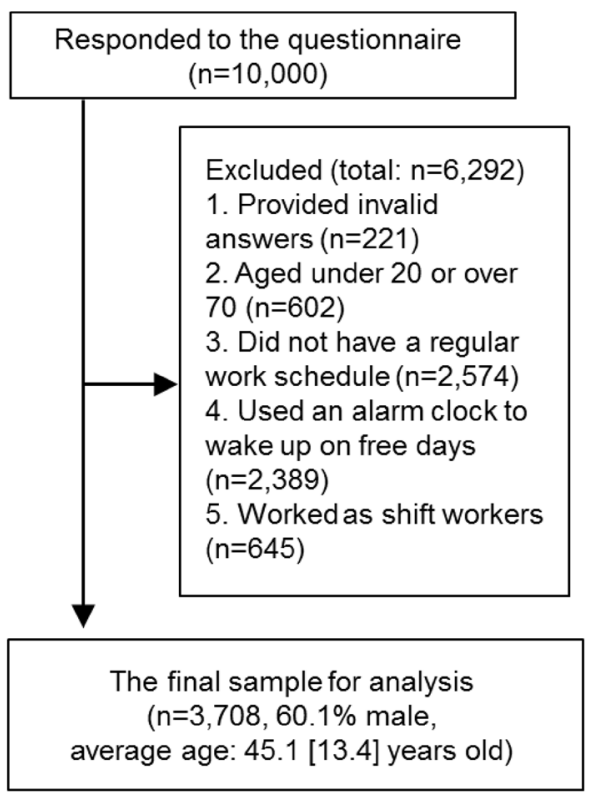


6 p.m. (min), and postal code of residence. The latter was translated into latitude and longitude.

To examine chronotypes, SJL, and other sleep variables, the Japanese version of the MCTQ was used [2,21]. The MCTQ included questions about shift work, the amount of time spent outdoors in daylight, the number of workdays and free days during the week, and bedtime and wake-up time including sleep/wake latency (in min) on workdays and on free days. Sleep duration across the week was calculated by averaging the sleep duration on workdays and free days. SJL was measured by subtracting each participant's midpoint of sleep on workdays (MSW) from their midpoint of sleep on free days (MSF). Both relative values and absolute values of SJL (SJLrel, SJLabs) were calculated. The chronotype, which is an individual's preference in sleep timing, was assessed using sleep-debt-corrected MSF (MSFsc) (see Roenneberg et al. [7]).

A two-way analysis of variance (ANOVA) (sex $\times$ age category) was used to evaluate MSFsc and SJLabs. The Bonferroni-Dunn test was used for post hoc analysis. Statistical analyses were performed using SPSS version 22 (SPSS Inc., Chicago, IL, USA), and the level of significance was set at $p<0.05$.

\section{Results}

\section{Sample characteristics}

A description of the sample $(n=3708)$ regarding sociodemographic and health-related variables is presented in Table 1 and that regarding sleep-related variables in Table 2. Average participant age was 45.1 [SD 13.4] years, and the proportion of female participants in the study sample was $39.9 \%$. The average sleep duration during the week, MSFsc, SJLrel, and SJLabs were 6.6 [1.1], 3.7 [1.4], 0.83 [0.96], and 0.91 [0.89], respectively.

\section{Age-related changes in chronotype}

Changes in MSFsc with age are shown in Fig. 2. The MSFsc progressively shifted to earlier times with advancing age. A two-way ANOVA showed a significant interaction between the effects of sex and age category on the MSFsc $[F(1,4)=3.9, p<0.01]$. There was a significant main effect of age category $[F(4,3698)=99.7, p<0.01]$, but no significant main effect of $\operatorname{sex}[F(1,3698)=1.8$, $p=0.33]$. Sex differences were not observed in participants between their twenties and their fifties, while male participants showed earlier chronotypes than female participants in their sixties.
Table 1 Sociodemographic and health-related variables $(n=3708)$

\begin{tabular}{ll}
\hline Variables & $n(\%)$, or mean [SD] \\
\hline Sociodemographic variables & \\
Sex & $2228(60.1)$ \\
Men & $1480(39.9)$ \\
Women & $45.1[13.4]$ \\
Age (years) & \\
Family & $3039(82.0)$ \\
Living with family & $669(18.0)$ \\
Living alone & \\
Health-related variables & $22.5[3.6]$ \\
Body mass index & $860(23.2)$ \\
Smoking & $2848(76.8)$ \\
Yes & \\
No & $1881(50.7)$ \\
Alcohol & $1827(49.3)$ \\
Yes & \\
No & \\
Physical activity (30 min on more than 2 days/week for at least \\
1 year) \\
Yes & $1170(31.6)$ \\
No & $2538(68.4)$ \\
Medical care (disease under treatment) & \\
Yes & $1252(33.8)$ \\
No & $2456(66.2)$ \\
\hline
\end{tabular}

Table 2 Sleep-related variables

\begin{tabular}{ll}
\hline MCTQ variables & Mean [SD] \\
\hline MSW & $3.21[1.24]$ \\
MSF & $4.05[1.54]$ \\
MSFsc & $3.70[1.44]$ \\
SJLrel & $0.83[0.96]$ \\
SJLabs & $0.91[0.89]$ \\
SDw & $6.40[1.14]$ \\
SDf & $7.25[1.34]$ \\
SDweek & $6.61[1.09]$ \\
SLOSS & $1.26[1.44]$ \\
\hline
\end{tabular}

$M S W$ midpoint of sleep on workdays, $M S F$ midpoint of sleep on free days, $M S F S c$ mid-sleep time on free days corrected for sleep debt, SJLrel relative social jetlag, SJLabs absolute social jetlag, SDw sleep duration on workdays, $S D f$ sleep duration on free days, $S D$ week sleep duration during the week, SLOSS sleep loss during the week

\section{Relationship between chronotypes and SJL}

The relationship between MSFsc and SJLabs is shown in Fig. 3. Chronotypes with an MSFsc of 2 a.m. had the lowest SJLabs value $(0.30 \mathrm{~h})$. When the MSFsc is later, SJLabs is higher. SJLabs also exists in the group with an MSFsc $\leq 1$ $(0.88 \mathrm{~h})$. 


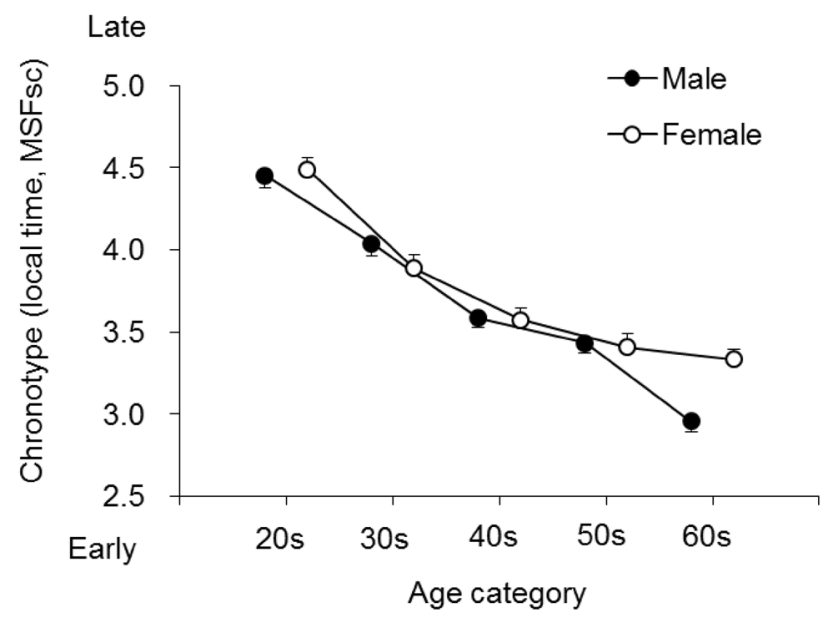

Fig. 2 Changes in chronotype with age. MSFsc mid-sleep time on free days corrected for sleep debt. Mean (SE)

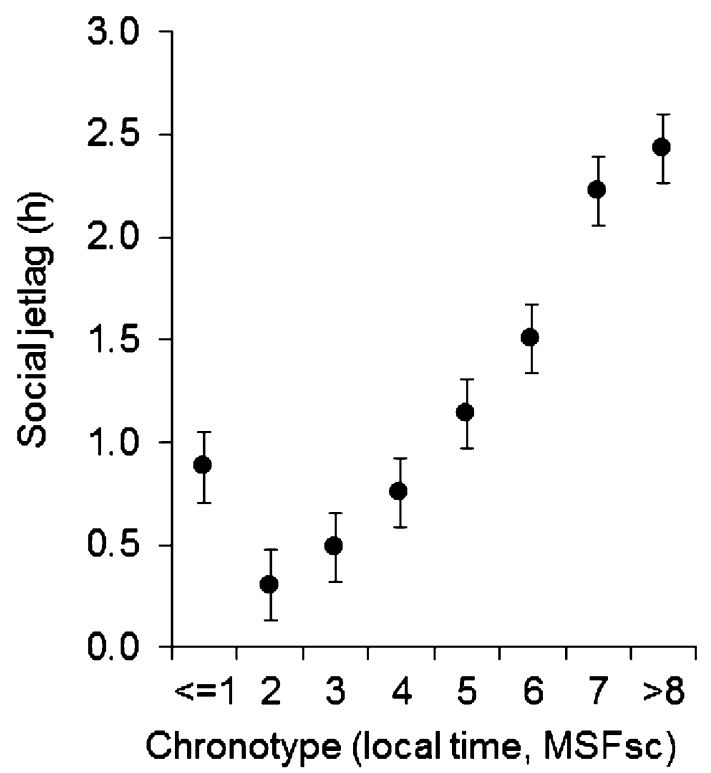

Fig. 3 Correlation between chronotype (MSFsc) and the absolute social jetlag

\section{Percentage of SJL in different age groups}

Table 3 shows the percentage of SJLrel and SJLabs in the different age categories. The percentage of participants with a negative SJLrel, indicating an earlier midpoint on free days than on weekdays, was only $3 \%$ in participants in their twenties and $6 \%$ overall. The average [SD] SJLabs was $0.91[0.89]$ hours, and the percentage of participants with an SJLabs of more than $1 \mathrm{~h}$ was $40.1 \%$. SJLabs became progressively lesser with advancing age. The percentage of participants with an SJLabs of more than $1 \mathrm{~h}$ was $61.0 \%$ in participants in their twenties, $53.2 \%$ in those in their thirties, and only $14.5 \%$ in those in their sixties.

A two-way ANOVA showed a significant main effect of age $[F(4,3698)=136.1, p<0.01]$, a main effect of sex $[F(1,3698)=4.4, p=0.04]$, and a significant interaction between the two $[F(1,4)=2.5, p=0.04]$. SJLabs was higher in female than in male participants. In particular, female participants showed a higher SJLabs than male participants in their thirties and forties (thirties: 1.22 vs $1.06 \mathrm{~h}$, forties: 1.04 vs $0.89 \mathrm{~h}$ ).

\section{Discussion}

This is the first nationwide, cross-sectional survey on SJL conducted in Asia. We conducted a web-based survey using the Japanese version of MCTQ in a total of 10,000 participants and presented the preliminary results based on the 3708 respondents. The key findings were (1) the average SJLabs was $54.6 \mathrm{~min}$ and the percentage of participants with an SJLabs of more than $1 \mathrm{~h}$ was $40.1 \%$; (2) SJLabs becomes progressively smaller with advancing age.

The mean MSFsc, a measure of chronotype, was 3.7 (3:42 a.m.), which is 32 min earlier than the average chronotype of MCTQ respondents from Germany, Switzerland, the Netherlands and Austria [7], and 37 min later than the average chronotype from the US population (using MSF) [18]. Chronotypes were found to shift to earlier times with advancing age (Fig. 2). This is in line with the results from the central European population [7], and from the US [18]. In the present study, we did not have enough data from participants in their teens to draw conclusions; it has, however, been reported that children are early chronotypes and that the MSFsc progressively shifts to later times throughout puberty and adolescence until it reaches a peak around the age of twenty [22].

The data from the central European population indicated the sex differences in chronotypes, that is, males have later chronotypes than females until around the age of menopause, and after that, males have earlier chronotypes than females [22]. However, no sex differences were observed in participants aged between the twenties and the fifties in this study sample. After the age of menopause, male participants showed earlier chronotypes than female participants, similar to the findings of previous studies [18, 22]. Further studies that include measurements of intrinsic circadian phase (e.g., melatonin secretion rhythm), and differences in lifestyle are warranted to investigate the question of sex differences.

SJLabs increased with the delay in MSFsc, consistent with an earlier report by Wittman [6]. To some extent, SJLabs also exists in the early chronotypes. While alarm clocks are the predominant cause for sleep loss in the later chronotypes, social pressure to stay up later than their 
Table 3 Prevalence of SJLrel (left) and SJLabs (right) in different age groups

\begin{tabular}{|c|c|c|c|c|c|c|}
\hline SJLrel & $20 \mathrm{~s}$ & $30 \mathrm{~s}$ & $40 \mathrm{~s}$ & $50 \mathrm{~s}$ & $60 \mathrm{~s}$ & All \\
\hline$<0 \mathrm{~h}(\%)$ & 3.3 & 5.1 & 6.7 & 6.3 & 8.6 & 6.1 \\
\hline $0-1 \mathrm{~h}(\%)$ & 37.0 & 43.0 & 52.0 & 63.5 & 77.3 & 54.9 \\
\hline $1-2 \mathrm{~h}(\%)$ & 35.2 & 36.7 & 29.8 & 24.8 & 13.4 & 27.8 \\
\hline $2-3 \mathrm{~h}(\%)$ & 18.5 & 11.0 & 9.3 & 3.4 & 0.6 & 8.4 \\
\hline$\geqq 3 \mathrm{~h}(\%)$ & 6.0 & 4.2 & 2.3 & 2.0 & 0.1 & 2.8 \\
\hline Mean (h) & 1.27 & 1.05 & 0.85 & 0.67 & 0.39 & 0.83 \\
\hline $\mathrm{SD}(\mathrm{h})$ & 1.15 & 1.02 & 0.92 & 0.88 & 0.52 & 0.96 \\
\hline SJLabs & $20 \mathrm{~s}$ & $30 \mathrm{~s}$ & $40 \mathrm{~s}$ & $50 \mathrm{~s}$ & $60 \mathrm{~s}$ & All \\
\hline$<0 \mathrm{~h}(\%)$ & - & - & - & - & - & - \\
\hline $0-1 \mathrm{~h}(\%)$ & 39.0 & 46.8 & 57.0 & 68.8 & 85.5 & 59.9 \\
\hline $1-2 \mathrm{~h}(\%)$ & 36.1 & 37.4 & 30.8 & 25.4 & 13.7 & 28.5 \\
\hline $2-3 \mathrm{~h}(\%)$ & 18.6 & 11.3 & 9.8 & 3.6 & 0.6 & 8.6 \\
\hline$\geqq 3 \mathrm{~h}(\%)$ & 6.3 & 4.5 & 2.4 & 2.3 & 0.1 & 3.0 \\
\hline Mean (h) & 1.36 & 1.13 & 0.95 & 0.76 & 0.43 & 0.91 \\
\hline SD (h) & 1.04 & 0.94 & 0.82 & 0.81 & 0.49 & 0.89 \\
\hline
\end{tabular}

SJLrel relative SJL, SJLabs absolute SJL

biological bedtime commonly causes sleep loss in early chronotypes [3]. SJL may be affected by not only late chronotype but also sleep debt on weekdays. From the UK study, participants with shorter sleep duration showed higher SJL [17]. Participants with late chronotype would not experience SJL if the work schedule permits enough sleep; for example, flextime schedule, late shift, etc.

The percentage of participants with a negative SJLrel, meaning an earlier sleep midpoint on weekends than on weekdays, was only $6 \%$ overall. It is not surprising that a large majority of the study population did not show earlier sleep timing on weekends, given that the intrinsic period of the human clock is longer than $24 \mathrm{~h}$ [23]. The mean value of SJLabs was $54.6 \mathrm{~min}$ overall. The percentages of participants with an SJLabs $\geq 1 \mathrm{~h}$ and SJLabs $\geq 2 \mathrm{~h}$ among our survey participants were $40.1 \%$ and $11.6 \%$, respectively. Much of the previous work on SJL has focused on specific subsets of the population, for example university students or young adults [6, 10, 24-26]. Thus, the current percentage estimates for the general population are limited. The percentage of SJLabs in the present study was somewhat lower than that found in a previous study on a central European population ( $\geq 1$ h: $69 \%$; $\geq 2$ h: $33 \%$ ) [3]. The results were close to the large Dutch sample ( $\geq 1 \mathrm{~h}$ SJL: 39\%) [27], and somewhat higher than the results of the Australian National Sleep Health Foundation Study ( $\geq 1$ h: $31 \%$; $\geq 2$ h: $10 \%$ ) [15] and the UK National Diet and Nutrition Survey (average SJL in participants who sleep less than $7 \mathrm{~h}$ and $7-8 \mathrm{~h}$ is $36 \mathrm{~min}$ and 12 min, respectively) [17].

Similar to the changes observed in the chronotype, which advanced gradually from early to late adulthood, SJLabs also decreased proportionally in the same age groups in this study. The percentages of participants with an SJLabs $\geq 1 \mathrm{~h}$ and SJLabs $\geq 2 \mathrm{~h}$ were $61.0 \%$ and $24.9 \%$ in their twenties, and $14.4 \%$ and $0.7 \%$ in their sixties, respectively. Early wake-up and bedtime schedules socially required, in particular among young people, may lead to health problems and deterioration of performance induced by the mismatch between biological and social time. As females show higher SJLabs than males, they might be at a higher risk for developing adverse health symptoms associated with SJL.

This study has some limitations. First, we used data from an internet-based survey in which subjects who were interested in their sleep health might have been more likely to participate. Second, the response rate was relatively low, which could cause response biases. Third, we used crosssectional data, and hence, we cannot provide information on the causality of the observed associations.

In conclusion, a considerable number of participants of our Japanese population, as represented in the current MCTQ survey, showed differences in sleep behavior between workdays and free days. Young people in particular commonly oversleep on free days to compensate for the sleep debt accumulated over the workweek because of their late chronotype. Thus, understanding the association of SJL and factors that contribute to an increased SJL risk may be important for disease prevention strategies targeting SJL. As the current preliminary study could not elucidate the associated factors, further studies, particularly longitudinal population studies of SJL targeting associated factors, long-term health, and work productivity outcomes would be needed. 


\section{Compliance with ethical standards}

Conflict of interest This study was supported in part by a Grant-in-Aid for Scientific Research from the Japan Society for the Promotion of Science (JSPS KAKENHI Grant Number 16K07545). Yoko Komada and Yuichi Inoue have received grant support from Merck \& Co., Inc., Eisai Co., Ltd, and Otsuka Pharmaceutical Co., Ltd. Isa Okajima has received lecture fees from Otsuka Pharmaceutical, MSD and Takeda Pharmaceutical, and research funding from NEC Solution Innovators, Ltd. Shingo Kitamura has received lecture fees from Otsuka Pharmaceutical Co., Ltd., and research funding from Boehringer Ingelheim Japan, Inc. and VELDT Inc.

Ethical approval This study was approved by the ethics committee of Tokyo Medical University (no. 3307).

Open Access This article is distributed under the terms of the Creative Commons Attribution 4.0 International License (http://creativeco mmons.org/licenses/by/4.0/), which permits unrestricted use, distribution, and reproduction in any medium, provided you give appropriate credit to the original author(s) and the source, provide a link to the Creative Commons license, and indicate if changes were made.

\section{References}

1. Roenneberg T, Merrow M. The network of time: understanding the molecular circadian system. Curr Biol. 2003;13:R198-207.

2. Roenneberg T, Wirz-Justice A, Merrow M. Life between clocks: daily temporal patterns of human chronotypes. J Biol Rhythms. 2003;18:80-90.

3. Roenneberg T, Kantermann T, Juda M, Vetter C, Allebrandt KV. Light and the human circadian clock. Handb Exp Pharmacol. 2013;(217):311-31.

4. Lane JM, Vlasac I, Anderson SG, Kyle SD, Dixon WG, Bechtold DA, et al. Genome-wide association analysis identifies novel loci for chronotype in 100,420 individuals from the UK Biobank. Nat Commun. 2016;7:10889.

5. Osland TM, Bjorvatn BR, Steen VM, Pallesen S. Association study of a variable-number tandem repeat polymorphism in the clock gene PERIOD3 and chronotype in Norwegian university students. Chronobiol Int. 2011;28:764-70.

6. Wittmann M, Dinich J, Merrow M, Roenneberg T. Social jetlag: misalignment of biological and social time. Chronobiol Int. 2006;23:497-509.

7. Roenneberg T, Kuehnle T, Juda M, Kantermann T, Allebrandt $\mathrm{K}$, Gordijn M, et al. Epidemiology of the human circadian clock. Sleep Med Rev. 2007;11:429-38.

8. Rutters F, Lemmens SG, Adam TC, Bremmer MA, Elders PJ, Nijpels G, et al. Is social jetlag associated with an adverse endocrine, behavioral, and cardiovascular risk profile? J Biol Rhythms. 2014;29:377-83.

9. Roenneberg T, Allebrandt KV, Merrow M, Vetter C. Social jetlag and obesity. Curr Biol. 2012;22:939-43.

10. Parsons MJ, Moffitt TE, Gregory AM, Goldman-Mellor S, Nolan PM, Poulton R, et al. Social jetlag, obesity and metabolic disorder: investigation in a cohort study. Int J Obes (Lond). 2015;39:842-8.

11. Wong PM, Hasler BP, Kamarck TW, Muldoon MF, Manuck SB. Social jetlag, chronotype, and cardiometabolic risk. J Clin Endocrinol Metab. 2015;100:4612-20.
12. Islam Z, Akter S, Kochi T, Hu H, Eguchi M, Yamaguchi M, et al. Association of social jetlag with metabolic syndrome among Japanese working population: the Furukawa Nutrition and Health Study. Sleep Med. 2018;51:53-8.

13. Levandovski R, Dantas G, Fernandes LC, Caumo W, Torres I, Roenneberg T, et al. Depression scores associate with chronotype and social jetlag in a rural population. Chronobiol Int. 2011;28:771-8.

14. Saitoh K, Furihata R, Kaneko Y, Suzuki M, Takahashi S, Uchiyama M. Association of serum BDNF levels and the BDNF Val66Met polymorphism with the sleep pattern in healthy young adults. PLoS One. 2018;13:e0199765.

15. Lang CJ, Reynolds AC, Appleton SL, Taylor AW, Gill TK, McEvoy RD, et al. Sociodemographic and behavioural correlates of social jetlag in Australian adults: results from the 2016 National Sleep Health Foundation Study. Sleep Med. 2018;51:133-9.

16. Gabud RS, Manalang GF, Chua RBL, Mendoza ER, LozanoKuhne JP. An assessment of chronotype and social jetlag among Filipinos. Int J Philipp Sci Technol. 2015;1:31-40.

17. Almoosawi S, Palla L, Walshe I, Vingeliene S, Ellis JG. Long sleep duration and social jetlag are associated inversely with a healthy dietary pattern in adults: results from the UK National Diet and Nutrition Survey Rolling Programme Y1-Y4. Nutrients. 2018. https://doi.org/10.3390/nu10091131

18. Fischer D, Lombardi DA, Marucci-Wellman H, Roenneberg T. Chronotypes in the US-influence of age and sex. PLoS One. 2017;12:e0178782.

19. Gradisar M, Gardner G, Dohnt H. Recent worldwide sleep patterns and problems during adolescence: a review and meta-analysis of age, region, and sleep. Sleep Med. 2011;12:110-8.

20. Hafner M, Stepanek M, Taylor J, Troxel WM, van Stolk C. Why sleep matters-the economic costs of insufficient sleep: a crosscountry comparative analysis. Rand Health Q. 2017;6:11.

21. Kitamura S, Hida A, Aritake S, Higuchi S, Enomoto M, Kato M, et al. Validity of the Japanese version of the Munich ChronoType Questionnaire. Chronobiol Int. 2014;31:845-50.

22. Foster RG, Roenneberg T. Human responses to the geophysical daily, annual and lunar cycles. Curr Biol. 2008;18:R784-94.

23. Czeisler CA, Duffy JF, Shanahan TL, Brown EN, Mitchell JF, Rimmer DW, et al. Stability, precision, and near-24-hour period of the human circadian pacemaker. Science. 1999;284:2177-81.

24. Diaz-Morales JF, Escribano C. Social jetlag, academic achievement and cognitive performance: understanding gender/sex differences. Chronobiol Int. 2015;32:822-31.

25. Haraszti RA, Ella K, Gyongyosi N, Roenneberg T, Kaldi K. Social jetlag negatively correlates with academic performance in undergraduates. Chronobiol Int. 2014;31:603-12.

26. McMahon DM, Burch JB, Wirth MD, Youngstedt SD, Hardin JW, Hurley TG, et al. Persistence of social jetlag and sleep disruption in healthy young adults. Chronobiol Int. 2018;35:312-28.

27. Koopman ADM, Rauh SP, van't Riet E, Groeneveld L, van der Heijden AA, Elders PJ, et al. The Association between social jetlag, the metabolic syndrome, and type 2 diabetes mellitus in the general population: the New Hoorn Study. J Biol Rhythms. 2017;32:359-68.

Publisher's Note Springer Nature remains neutral with regard to jurisdictional claims in published maps and institutional affiliations. 